\title{
Crossing numbers
}

\author{
János Pach* \\ Courant Institute, New York University \\ and Hungarian Academy of Sciences
}

\begin{abstract}
The crossing number of a graph $G$ is the minimum number of crossings in a drawing of $G$. The determination of the crossing number is an NP-complete problem. We present two general lower bounds for the crossing number, and survey their applications and generalizations.
\end{abstract}

\section{Preliminaries}

A drawing of a graph $G$ is a representation of $G$ in the plane such that its vertices are represented by distinct points and its edges by simple continuous arcs connecting the corresponding point pairs. If it is clear whether we talk about the "abstract" graph $G$ or its planar representation, these points and arcs will also be called vertices and edges, respectively. For simplicity, we assume that in a drawing (a) no edge passes through any vertex other than its endpoints, (b) no two edges touch each other (i.e., if two edges have a common interior point, then at this point they properly cross each other), and (c) no three edges cross at the same point.

Turán [26] defined the crossing number of $G, \mathrm{CR}(G)$, as the smallest number of edge crossings in any drawing of $G$. Clearly, $\mathrm{CR}(G)=0$ if and only if $G$ is planar. According to a famous conjecture of Zarankiewicz [10], the crossing number of the complete graph $K_{n, m}$ with $n$ and $m$ vertices in its classes satisfies

$$
\mathrm{CR}\left(K_{n, m}\right)=\left\lfloor\frac{m}{2}\right\rfloor \cdot\left\lfloor\frac{m-1}{2}\right\rfloor \cdot\left\lfloor\frac{n}{2}\right\rfloor \cdot\left\lfloor\frac{n-1}{2}\right\rfloor .
$$

Kleitman [11] verified this conjecture in the special case $\min \{m, n\} \leq 6$, and Woodall [29] for $\min \{m, n\}=7$.

Garey and Johnson [9] proved that the determination of the crossing number is an $N P$-complete problem. In the past twenty years, it turned out that crossing numbers play an important role in various fields of discrete and computational geometry, and they can also be used, e.g.,to obtain lower bounds on the chip area required for the VLSI circuit layout of a graph [12]. In this lecture, first we recall two important general results for crossing numbers, and outline some of their applications. Next we describe some recent extensions and generalizations of these theorems. After that we introduce three alternative notions of crossing number, and analyze their relationship. Finally, we state some tantalizing open problems.

\footnotetext{
* Supported by the National Science Foundation (USA) and the National Fund for Scientific Research (Hungary). e-mail: pach@cims.nyu.edu
} 


\section{Two Important Bounds and Their Applications}

The following result was proved by Ajtai-Chvátal-Newborn-Szemerédi and, independently, by Leighton. The best known constant, $1 / 33.75$, in the theorem is due to Pach and Tóth.

Theorem 2.1. [2],[12],[19] For any graph $G$ with $n$ vertices and $e \geq 7.5 n$ edges, we have

$$
\mathrm{CR}(G) \geq \frac{1}{33.75} \frac{e^{3}}{n^{2}}
$$

and this estimate is tight up to a constant factor.

To prove the tightness, let $n \ll e \ll n^{2}$, and consider a graph, whose vertex set is a $\lfloor\sqrt{n}\rfloor$ by $\lfloor\sqrt{n}\rfloor$ piece of the integer grid in the plane, and connect two gridpoints, $p$ and $q$, by an edge if and only if their distance satisfies

$$
|p-q| \leq \sqrt{\frac{2}{\pi} \cdot \frac{e}{n}}
$$

It is not hard to show that the crossing number of this graph is at most $0.06 e^{3} / n^{2}$.

To state the second general bound on the crossing number, we need a definition. Let $G$ be a graph with vertex set $V(G)$ and edge set $E(G)$. The bisection width of $G, b(G)$, is defined as the minimum number of edges, whose removal splits the graph into two roughly equal subgraphs. More precisely, $b(G)$ is the minimum number of edges running between $V_{1}$ and $V_{2}$, over all partitions of the vertex set of $G$ into two disjoint parts $V_{1} \cup V_{2}$ such that $\left|V_{1}\right|,\left|V_{2}\right| \geq|V(G)| / 3$.

Leighton observed that there is an intimate relationship between the bisection width and the crossing number of a graph [13], which is based on the LiptonTarjan separator theorem for planar graphs [14]. The following version of this relationship was obtained by Pach, Shahrokhi, and Szegedy.

Theorem 2.2. [12],[16] Let $G$ be a graph of $n$ vertices with degrees $d_{1}, d_{2}, \ldots, d_{n}$. Then

$$
b(G) \leq 10 \sqrt{\mathrm{CR}(G)}+2 \sqrt{\sum_{i=1}^{n} d_{i}^{2}} .
$$

We briefly mention three applications of Theorem 2.1 and three applications of Theorem 2.2. The first two applications are due to L. Székely, the third to T. Dey.

Applications. 2.1.a. Szemerédi-Trotter theorem. [25], [5],[15] Given $n$ points and $m$ lines in the Euclidean plane, the number of incidences between them is at most $2.57 m^{2 / 3} n^{2 / 3}+m+n$.

2.1.b. Spencer-Szemerédi-Trotter theorem. [24] The number of unit distances determined by $n$ points in the plane is $O\left(n^{4 / 3}\right)$.

A family $\Gamma$ of curves in the plane is said to have $d$ degrees of freedom, if there exists an integer $s$ such that

(a) no two curves in $\Gamma$ have more than $s$ points in common, and 
(b) for any $d$ points, there are at most $s$ curves in $\Gamma$ passing through all of them.

Pach and Sharir [17] applied Theorem 2.1 to obtain the following common generalization of the last two theorems (2.1.a-b). Let $\Gamma$ be a family of curves in the plane with $d$ degrees of freedom. Then the maximum number of incidences between $n$ points in the plane and $m$ elements of $\Gamma$ is

$$
O\left(n^{d /(2 d-1)} m^{(2 d-2) /(2 d-1)}+n+m\right) .
$$

2.1.c. Dey theorem. [6] Given a set of $2 n$ points in the plane, a line connecting two of them is called a halving line if there are precisely $n-1$ points on both of its sides. The number of halving lines for a set of $2 n$ points in general position in the plane is $O\left(n^{4 / 3}\right)$.

2.2.a. For a random graph $G$ with $n$ vertices and $e \geq 4 n$ edges, we almost surely have $\mathrm{CR}(G) \geq c e^{2}$, for some positive constant $c$.

2.2.b. [16],[1] Let $G$ be any (so-called geometric) graph with $n$ vertices, drawn in the plane so that its edges are represented by straight-line segments. Suppose that $G$ has no $k$ pairwise disjoint edges $(k>2)$. Then there exists a constant $c_{k}$ depending only on $k$ such that $G$ has at most $c_{k} n \log ^{2 k-6} n$ edges. Valtr [28] has recently reduced the exponent $2 k-6$ to 1 , for every $k>3$, but it is possible that one can completely get rid of the logarithmic factor.

2.2.c. [21] For every $n$, there exist a planar graph $G_{n}$ of $n$ vertices and an assignment of locations (distinct points) for the vertices such that in any planar drawing of $G_{n}$ with the property that each vertex is mapped into the point assigned to it and each edge is represented by a polygonal path, there are at least $n / 100$ edges consisting of at least $n / 100$ segments.

\section{Extensions and Generalizations}

Let $K(n, e)$ denote the minimum crossing number of a graph $G$ with $n$ vertices and $e$ edges. That is,

$$
K(n, e)=\min \{\mathrm{CR}(G):|V(G)|=n,|E(G)|=e\} .
$$

It follows from Theorem 2.1 that, for $e \geq 4 n, K(n, e) n^{2} / e^{3}$ is bounded from below and from above by two positive constants. Erdős and Guy [7] conjectured that if $e \gg n$ (i.e., if $\lim _{n \rightarrow \infty} f(n) / g(n)=\infty$ ), then $\lim K(n, e) n^{2} / e^{3}$ exists. This conjecture is almost true.

Theorem 3.1. [18] If $n \ll e \ll n^{2}$, then

$$
\lim _{n \rightarrow \infty} \frac{K(n, e)}{e^{3} / n^{2}}=K_{0}
$$

exists. 
Analogously, for every $g \geq 0$, one can define a new crossing number, $\mathrm{CR}_{g}(G)$, as the minimum number of crossings in any drawing of $G$ on the torus with $g$ holes. Let

$$
K_{g}(n, e)=\min \left\{\mathrm{CR}_{g}(G):|V(G)|=n,|E(G)|=e\right\} .
$$

Theorem 3.2. [18] For fixed $g \geq 0$, if $n \ll e \ll n^{2}$, then

$$
\lim _{n \rightarrow \infty} \frac{K(n, e)}{e^{3} / n^{2}}=K_{g}
$$

exists. Moreover, $K_{g}=K_{0}$ for every $g$.

Miklós Simonovits suggested that much stronger estimates may be valid for the crossing number(s), if we restrict our attention to some special classes of graphs, e.g., to graphs not containing some fixed, so-called forbidden subgraph. It turns out that this is indeed the case.

A graph property $\mathcal{P}$ is said to be monotone if for any graph $G$ satisfying $\mathcal{P}$, every subgraph of $G$ also satisfies $\mathcal{P}$. For any monotone property $\mathcal{P}$, let $\operatorname{ex}(n, \mathcal{P})$ denote the maximum number of edges that a graph of $n$ vertices can have if it satisfies $\mathcal{P}$. In the special case when $\mathcal{P}$ is the property that the graph does not contain a subgraph isomorphic to a fixed forbidden subgraph $H$, we write $\operatorname{ex}(n, H)$ for $\operatorname{ex}(n, \mathcal{P})$.

Theorem 3.3. [18] Let $\mathcal{P}$ be a monotone graph property with $\operatorname{ex}(n, \mathcal{P})=O\left(n^{1+\alpha}\right)$ for some $\alpha>0$.

Then there exist two constants $c, c^{\prime}>0$ such that the crossing number of any graph $G$ with property $\mathcal{P}$, which has $n$ vertices and $e \geq c n \log ^{2} n$ edges, satisfies

$$
\mathrm{CR}(G) \geq c^{\prime} \frac{e^{2+1 / \alpha}}{n^{1+1 / \alpha}} .
$$

This bound is asymptotically tight, up to a constant factor.

In some interesting special cases when we know the precise order of magnitude of the function $\operatorname{ex}(n, \mathcal{P})$, we obtain some slightly stronger results. The girth of a graph is the length of its shortest cycle.

Theorem 3.4. [18] Let $G$ be a graph with $n$ vertices and $e \geq 4 n$ edges, whose girth is larger than $2 r$.

Then the crossing number of $G$ satisfies

$$
\mathrm{CR}(G) \geq c_{r} \frac{e^{r+2}}{n^{r+1}}
$$

where $c_{r}>0$ is a suitable constant. For $r=2,3$, and 5 , these bounds are asymptotically tight, up to a constant factor.

Theorem 3.5. Let $G$ be a graph with $n$ vertices and $e \geq 4 n$ edges, which does not contain a complete bipartite subgraph $K_{r, s}$ with $r$ and $s$ vertices in its classes, $s \geq r$. 
Then the crossing number of $G$ satisfies

$$
\mathrm{CR}(G) \geq c_{r, s} \frac{e^{3+1 /(r-1)}}{n^{2+1 /(r-1)}}
$$

where $c_{r, s}>0$ is a suitable constant. These bounds are tight up to a constant factor, whenever $s>(r-1)$ !.

To see that the order of magnitude of the estimate in Theorem 3.5 cannot be improved, for $r=s=2$ (say), take a $K_{2,2}$-free graph $H$ with $(2 e / n)^{2}$ vertices of degree $2 e / n$. Let $G$ be the union of $n^{3} /\left(4 e^{2}\right)$ pairwise disjoint components isomorphic to $H$. Using the trivial estimate $\mathrm{CR}(H) \leq\left(\begin{array}{c}|E(H)| \\ 2\end{array}\right)$, we obtain that

$$
\mathrm{CR}(G) \leq \frac{n^{3}}{4 e^{2}} \mathrm{CR}(H) \leq \frac{n^{3}}{4 e^{2}}\left(\begin{array}{c}
4 e^{3} / n^{3} \\
2
\end{array}\right) \leq \frac{2 e^{4}}{n^{3}}
$$

as required.

The basic tool for the proof of most of the results in this section is Theorem 2.2 , and its generalization to tori.

\section{Three Other Crossing Numbers}

We define three variants of the notion of crossing number.

(1) The rectilinear crossing number, LIN-CR $(G)$, of a graph $G$ is the minimum number of crossings in a drawing of $G$, in which every edge is represented by a straight-line segment.

(2) The pairwise crossing number of $G$, PAIR-CR $(G)$, is the minimum number of crossing pairs of edges over all drawings of $G$. (Here the edges can be represented by arbitrary continuous curves, so that two edges may cross more than once, but every pair of edges can contribute at most one to PAIR-CR $(G)$.)

(3) The odd-crossing number of $G$, ODD-CR $(G)$, is the minimum number of those pairs of edges which cross an odd number of times, over all drawings of $G$.

It readily follows from the definitions that

$$
\operatorname{ODD}-\mathrm{CR}(G) \leq \operatorname{PAIR-CR}(G) \leq \mathrm{CR}(G) \leq \operatorname{LIN}-\mathrm{CR}(G) .
$$

Bienstock and Dean [3] exhibited a series of graphs with crossing number 4, whose rectilinear crossing numbers are arbitrary large. However, we cannot rule out the possibility that

$$
\operatorname{ODD}-\mathrm{CR}(G)=\operatorname{PAIR-CR}(G)=\mathrm{CR}(G),
$$

for every graph $G$. This is perhaps the most exciting open problem in the area.

The determination of the odd-crossing number can be rephrased as a purely combinatorial problem, thus the possible coincidence of the above three crossing numbers would offer a spark of hope that there exists an efficient approximation algorithm for computing their value. 
According to a remarkable theorem of Hanani (alias Chojnacki) [4] and Tutte [27], if a graph $G$ can be drawn in the plane so that any pair of its edges cross an even number of times, then it can also be drawn without any crossing. In other words, ODD-CR $(G)=0$ implies that $\mathrm{CR}(G)=0$. Note that in this case, by a theorem of Fáry [8], we also have that LIN-CR $(G)=0$.

The main difficulty in this problem is that a graph has so many essentially different drawings that the computation of any of the above crossing numbers, for a graph of only 15 vertices, appears to be a hopelessly difficult task even for a very fast computer [22].

Theorem 4.1. [9],[20] Given a graph $G$ and an integer $K$, it is an NP-complete problem to decide whether any of the above crossing numbers of $G$ is at most $K$.

All we can show is that the parameters $\mathrm{CR}(G), \operatorname{PAIR}-\mathrm{CR}(G)$, and ODD-CR $(G)$, are not completely unrelated.

Theorem 4.2. [20] For any graph $G$, we have

$$
\mathrm{CR}(G) \leq 2(\mathrm{ODD}-\mathrm{CR}(G))^{2} .
$$

The proof of the last statement is based on the following sharpening of the Hanani-Tutte Theorem.

Theorem 4.3. [20] An arbitrary drawing of any graph in the plane can be redrawn in such a way that no edge, which originally crossed every other edge an even number of times, would participate in any crossing.

\section{References}

1. P. Agarwal, B. Aronov, J. Pach, R. Pollack, and M. Sharir: Quasi-planar graphs have a linear number of edges, Combinatorica 17 (1997), 1-9.

2. M. Ajtai, V. Chvátal, M. Newborn, E. Szemerédi: Crossing-free subgraphs, Annals of Discrete Mathematics 12 (1982), 9-12.

3. D. Bienstock and N. Dean: Bounds for rectilinear crossing numbers, Journal of Graph Theory 17 (1993), 333-348.

4. Ch. Chojnacki (A. Hanani): Über wesentlich unplättbare Kurven im dreidimensionalen Raume, Fund. Math. 23 (1934), 135-142.

5. K. Clarkson, H. Edelsbrunner, L. Guibas, M. Sharir, E. Welzl: Combinatorial complexity bounds for arrangements of curves and surfaces, Discrete and Computational Geometry 5 (1990), 99-160.

6. T. K. Dey: Improved bounds for planar $k$-sets and related problems, Discrete and Computat. Geometry 19 (1998), 373-382.

7. P. Erdős and R. K. Guy: Crossing number problems, American Mathematical Monthly 80 (1973), 52-58.

8. I. Fáry: On straight line representation of planar graphs, Acta Univ. Szeged. Sect. Sci. Math. 11 (1948), 229-233.

9. M. R. Garey and D. S. Johnson: Crossing number is NP-complete, SIAM Journal of Algebraic and Disccrete Methods 4 (1983), 312-316. 
10. R. K. Guy: The decline and fall of Zarankiewicz's theorem, in: Proof Techniques in Graph Theory, Academic Press, New York, 1969, 63-69.

11. D. J. Kleitman: The crossing number of $K_{5, n}$, Journal of Combinatorial Theory 9 (1970), 315-323.

12. T. Leighton: Complexity Issues in VLSI, Foundations of Computing Series, MIT Press, Cambridge, MA, 1983.

13. F. T. Leighton: New lower bound techniques for VLSI, Math. Systems Theory 17 (1984), 47-70.

14. R. Lipton and R. Tarjan: A separator theorem for planar graphs, SIAM J. Applied Mathematics 36 (1979), 177-189.

15. J. Pach and P.K. Agarwal: Combinatorial Geometry, J. Wiley and Sons, New York, 1995.

16. J. Pach, F. Shahrokhi, and M. Szegedy: Applications of the crossing number, Algorithmica 16 (1996), 111-117.

17. J. Pach and M. Sharir: On the number of incidences between points and curves, Combinatorics, Probability and Computing 7 (1998), 121-127.

18. J. Pach, J. Spencer, and G. Tóth: New bounds on crossing numbers, 15th ACM Symposium on Computational Geometry, 1999, 124-133. Also in: Discrete and Computational Geometry, accepted.

19. J. Pach and G. Tóth: Graphs drawn with few crossings per edge, Combinatorica 17 (1997), 427-439.

20. J. Pach and G. Tóth: Which crossing number is it, anyway?, in: Proceedings of 39th Annual Symposium on Foundations of Computer Science, Palo Alto, 1998, 617-626.

21. J. Pach and R. Wenger: Embedding planar graphs with fixed vertex locations, in: Graph Drawing '98 (Sue Whitesides, ed.), Lecture Notes in Computer Science, Springer-Verlag, Berlin, 1999, to appear.

22. R. B. Richter and C. Thomassen: Relations between crossing numbers of complete and complete bipartite graphs, American Mathematical Monthly, February 1997, 131-137.

23. L. A. Székely: Crossing numbers and hard Erdős problems in discrete geometry, Combinatorics, Probability and Computing 6 (1998), 353-358.

24. J. Spencer, E. Szemerédi, and W. T. Trotter: Unit distances in the Euclidean plane, in: Graph Theory and Combinatorics (B. Bollobás, ed.), Academic Press, New York, 1984, 293-303.

25. E. Szemerédi and W. T. Trotter: Extremal problems in discrete geometry, Combinatorica 3 (1983), 381-392.

26. P. Turán: A note of welcome, Journal of Graph Theory 1 (1977), 7-9.

27. W. T. Tutte: Toward a theory of crossing numbers, Journal of Combinatorial Theory 8 (1970), 45-53.

28. P. Valtr: On geometric graphs with no $k$ pairwise parallel edges, Discrete and Computational Geometry 19 (1998), 461-469.

29. D. R. Woodall: Cyclic-order graphs and Zarankiewicz's crossing-number conjecture, Journal of Graph Theory 17 (1993), 657-671.

This article was processed using the $\mathrm{AT}_{\mathrm{E}} \mathrm{X}$ macro package with LLNCS style 